\title{
Synthesis, Spectral and Antimicrobial Studies of Bis (Cyclopentadienyl) Titanium (IV) Bis (0,0'-Dialkyl and Alkylenedithiophosphate) Complexes
}

\author{
Adnan A. S. El Khaldy ${ }^{1}$, Florence Okafor ${ }^{2}$, Alaa M. Abu Shanab ${ }^{3}$ \\ ${ }^{1}$ Department of Physics, Chemistry, and Mathematics, College of Engineering, Technology and Physical \\ Sciences, Alabama A\&M University, Normal, AL, USA \\ ${ }^{2}$ Department of Biological \& Environmental Sciences, College of Agricultural, Life and Natural Sciences, \\ Alabama A\&M University, Normal, AL, USA \\ ${ }^{3}$ Chemistry Department, Al-Aqsa University, Gaza, Palestine \\ Email: ${ }^{*}$ adnan.elkhaldy@aamu.edu
}

Received 14 October 2014; revised 30 November 2014; accepted 15 December 2014

Copyright (C) 2014 by authors and Scientific Research Publishing Inc.

This work is licensed under the Creative Commons Attribution International License (CC BY).

http://creativecommons.org/licenses/by/4.0/

c) (i) Open Access

\section{Abstract}

A new complexes of $\mathrm{Cp}_{2} \mathrm{Ti}\left[\mathrm{S}_{2} \mathrm{P}(\mathrm{OR})_{2}\right]_{2}$ (where $\mathrm{R}=\mathrm{Et}$, Pr-n, Pr-i, Bu-i and $\mathrm{Ph}$ ) and $\mathrm{Cp}_{2} \mathrm{Ti}\left[\mathrm{S}_{2} \mathrm{POGO}\right]_{2}$ (where $\mathrm{G}=-\mathrm{CH}_{2} \mathrm{CMe}_{2} \mathrm{CH}_{2-}^{-},-\mathrm{CH}_{2} \mathrm{CEt}_{2} \mathrm{CH}_{2}$ - and $-\mathrm{CMe}_{2} \mathrm{CMe}_{2}-$ ) were prepared by the dropwise addition of the appropriate $0,0^{\prime}$-dialkyl or -alkylenedithiophosphoric acid to biscyclopentadienyl titanium dichloride in 1:2 molar ratio and refluxed in benzene solution. These novel deep red colored complexes were characterized by elemental analyses, molecular weight measurements and spectroscopic techniques (IR., NMR ${ }^{1} \mathrm{H},{ }^{13} \mathrm{C}$ and ${ }^{31} \mathrm{P}$ NMR). These titanium (IV) dithio complexes have also been screened for their antibacterial activities.

\section{Keywords}

Titanium (IV) Dialkyl and Alkylenedithiophosphate Complexes

\section{Introduction}

The synthesis of coordination compounds with sulfur containing ligands has been in the center of interest in chemical research for many years [1]-[6]. A survey of literature on dithiophosphato derivatives of titanium and

${ }^{*}$ Corresponding author.

How to cite this paper: El Khaldy, A.A.S., Okafor, F. and Shanab, A.M.A. (2014) Synthesis, Spectral and Antimicrobial Studies of Bis (Cyclopentadienyl) Titanium (IV) Bis (O,O'-Dialkyl and Alkylenedithiophosphate) Complexes. International Journal of Organic Chemistry, 4, 339-346. http://dx.doi.org/10.4236/ijoc.2014.45037 
organotitanium reveals that only simple derivatives (e.g., those containing organic and halo substituents on titanium on addition to the dithiophosphato group) have been described [7] [8]. Derivatives containing other monodentate ligands in addition to dithiophosphate have not been isolated. Dialkyl and alkylenedithiophosphates exhibit a variety of coordination modes of bonding [9]-[12] and their metal complexes have important biochemical, analytical and industrial applications [13]-[17]. The biocidial importance of organophosphorus compounds is well known. The synthesis of organotitanium (IV) dithiophosphate compounds provide model systems of interest because the presence of biologically active organophosphorus and organo titanium moieties in a single molecule could provide new information about the bioactivity of titanium compound. In view of the ready conversion of Ti-Cl bonded into Ti-S bonded compounds, the preparative route chosen for the above compounds was the direct interaction between Biscyclopentadienyl Titanium (IV) dichloride and dialkyl (or alkylene) diothiophosphoric acids. Thus, the reactions of Titanocene with dithiophosphoric acids have been carried out in 1:2 molar ratios under mild condition.

Before discussing the results of the above reactions, it may be relevant to mention the structural features of the $\mathrm{Cp}_{2} \mathrm{TiCl}_{2}$ [18]. Titanocene does not adopt the typical "sandwich" structure like ferrocene due to the 4 ligands around the metal center, but rather takes on a distorted tetrahedral shape [19]. Although its crystal structure has not been determined, its structure has been demonstrated in a number of other organotitanium dithiophosphates. In dilute solution, however, the titanocene structure which is monomeric species containing unidentate dithiophosphates and 4-coordinated titanium atoms.

\section{Experimental}

Stringent precautions were taken to exclude moisture. Solvents (benzene, $n$-hexane) were dried by standard methods. Glycols were distilled before use; Titanocene (Merck) was used as received. Dialkyl and alkylenedithiophosphoric acids were prepared by the reaction of phosphorus pentasulfide and alcohols in a 1:4 ratio, and in a 1:2 ratio with glycols as described in the literature [20]. Sulfur was determined by Messenger's method as barium sulfate. Titanium was determined titanium oxide (cupferron method). Infrared spectra were recorded as Nujol mulls using CsI cells in the region $4000-200 \mathrm{~cm}^{-1}$ on an FT-IR 8201PC spectrophotometer.

${ }^{1} \mathrm{H}$ and ${ }^{13} \mathrm{C}$ spectra were recorded on a Jeol-FT NMR spectrometer-LA300 and using TMS as the internal reference. ${ }^{31} \mathrm{P}$ NMR spectra were recorded in $\mathrm{CHCl}_{3}$ using $\mathrm{H}_{3} \mathrm{PO}_{4}$ as an external reference on the same instrument. The following synthetic details for a specific 1:2 reaction represent the procedure used to synthesize all compounds.

\subsection{Reaction between Biscyclopentadienyl Titanium Dichloride with Dialkyl (OPr-n) and Alkylenedithiophosphoric Acids in 1:2 Molar Ratios}

A benzene $(\sim 10 \mathrm{ml})$ solution of $\mathrm{HS}_{2} \mathrm{P}(\mathrm{OPr}-\mathrm{n})_{2}(0.818 \mathrm{~g} ; 3.82 \mathrm{mmol})$ was added to benzene $(\sim 15 \mathrm{ml})$ solution of $\mathrm{Cp}_{2} \mathrm{TiCl}_{2}(0.475 \mathrm{~g} ; 1.91 \mathrm{mmol})$ dropwise with stirring at room temperature. The reaction mixture was refluxed for $\sim 5$ hour, during which the color of the reaction mixture changed from color red to dark red. The excess solvent was removed under reduced pressure and the product washed repeatedly by $n$-hexane and the desired product was finally dried under reduced pressure.

\subsection{Antimicrobial Studies}

Bioactivity studies were conducted using three bacterial strains; Escherichia coli, Bacillus cereus, and Pseudomonas aeruginosa as test microorganisms.

Susceptibility of the microorganisms to the novel chemical compounds was determined using the Agar diffusion method and in accordance with the CLSI (formerly NCCLS) guidelines [21]. This method was used as a qualitative method to determine whether each bacterium is resistant, intermediately resistant or susceptible to the synthesized chemicals. The broth cultures of the microorganisms were first grown until they had an optical density (OD) or absorbance of $0.8-1.0$ at a $600 \mathrm{~nm}$ wavelength. Inoculum of approximately $10^{6}$ colony forming unit (CFU) of each isolate was plated on to the Mueller-Hinton Agar plates to form a confluent or lawn growth. The test chemical (400 ug/ml) or control (70\% ethanol, v/v) was applied to sterile disks and placed on the inoculated plates and incubated at $37^{\circ} \mathrm{C}$ for $24 \mathrm{~h}$.

The zones of inhibition/clearance of microbial growth around the disks containing the extracts/control were 
measured. The zone of inhibition was defined as the shortest distance (in $\mathrm{mm}$ ) from the outside margin of the initial point of microbial growth. Three replicates were made for each test organism.

\section{Results and Discussion}

Biscyclopentadienyl titanium bis (dialkyl and alkylenedithiophosphate) have been synthesized by the reaction of biscyclopentadienyl titanium dichloride (Titanocene) with dialkyl and alkylenedithiophosphoric acids in 1:2 molar ratios in refluxing benzene as in Equations (1) and (2).

$$
\mathrm{Cp}_{2} \mathrm{TiCl}_{2}+2 \mathrm{HS}_{2} \mathrm{P}(\mathrm{OR})_{2} \rightarrow \mathrm{Cp}_{2} \mathrm{Ti}\left[\mathrm{S}_{2} \mathrm{P}(\mathrm{OR})_{2}\right]_{2}+2 \mathrm{HCl}
$$

where R = Et, Pr-n, Pr-i, Bu-i and Ph

$$
\mathrm{Cp}_{2} \mathrm{TiCl}_{2}+2 \mathrm{HS}_{2} \stackrel{\mathrm{POGO}}{\rightarrow} \rightarrow \mathrm{Cp}_{2} \mathrm{Ti}\left[\mathrm{S}_{2}{ }_{\mathrm{POGO}}\right]_{2}+2 \mathrm{HCl}
$$

$\mathrm{G}=-\mathrm{CH}_{2} \mathrm{CMe}_{2} \mathrm{CH}_{2}-,-\mathrm{CH}_{2} \mathrm{CEt}_{2} \mathrm{CH}_{2}$ - and $-\mathrm{CMe}_{2} \mathrm{CMe}_{2}$.

The color of the reaction medium changed from red to deep red color with the progress of reaction. Biscyclopentadienyl titanium bis (dialkyl and alkylenedithiophosphate) derivatives are deep red color solids in open and cyclic chain complexes. All these compounds are soluble in common organic solvents like benzene, dichloromethane and chloroform. The molecular weight of all these products determined by cryoscopic method in benzene indicated the monomeric nature of these products (Table 1).

\subsection{IR Spectra}

IR spectra of biscyclopentadienyl titanium bis (dialkyl and alkylenedithiophosphate), have been recorded in the region $4000-400 \mathrm{~cm}^{-1}$ [22] [23]. The band shown by the parent acids in the region $2544-2400 \mathrm{~cm}^{-1}$, due to SH stretching vibration, are absent for biscyclopentadienyl titanium bis(dialkyl and alkylenedithiophosphate) derivatives, indicating the formation of Ti-S bond with the appearance a new band in the regions $428-400 \mathrm{~cm}^{-1}$ [22] [24]. The bands present in the region $1104.0-1014.5 \mathrm{~cm}^{-1}$ and $937.3-800 \mathrm{~cm}^{-1}$ have been assigned to $v$ (P)-O-C and $v$ P-O-(C) stretching vibrations respectively. Strong bands in the region $995-921.9 \mathrm{~cm}^{-11}$ are due to dioxaphospholane and dioxaphosphorinane ring vibrations [25]-[27]. The bands observed present in the region 704.0- $638.0 \mathrm{~cm}^{-11}$ can be assigned to $v \mathrm{P}=\mathrm{S}$ vibrations [28]. The bands in medium intensities in the region 602 $513.0 \mathrm{~cm}^{-11}$ may be attributed to vibration of $\nu \mathrm{P}-\mathrm{S}$ asymmetric and symmetric vibrations [29]. Details regarding the individual bands have been included in Table 2.

Table 1. Physical properties and analytical data of biscyclopentadienyl titanium bis (O,O-dialkyl and alkylene dithiophosphate)

\begin{tabular}{|c|c|c|c|c|c|c|c|c|}
\hline SI. No. & Compounds & $\begin{array}{l}\text { Physical } \\
\text { State }\end{array}$ & M. P. ${ }^{\circ} \mathrm{C}$ & $\begin{array}{c}\text { Mol. Wt. } \\
\text { Found/(Calc.) }\end{array}$ & $\begin{array}{c}\% \mathrm{H} \\
\text { Found/(Calc.) }\end{array}$ & $\begin{array}{c}\% \mathrm{C} \\
\text { Found/(Calc.) }\end{array}$ & $\begin{array}{c}\text { \% S } \\
\text { Found/(Calc.) }\end{array}$ & $\begin{array}{c}\% \mathrm{Ti} \\
\text { Found/(Calc.) }\end{array}$ \\
\hline 1 & $\mathrm{Cp}_{2} \mathrm{Ti}\left[\mathrm{S}_{2} \mathrm{P}(\mathrm{OEt})_{2}\right]_{2}$ & $\begin{array}{c}\text { Deep } \\
\text { Red Solid }\end{array}$ & $137^{\circ}$ & $542.22 /(548.50)$ & $5.54 /(5.51)$ & 38.99/(39.41) & 22.43 / (23.38) & 7.10/(8.72) \\
\hline 2 & $\mathrm{Cp}_{2} \mathrm{Ti}\left[\mathrm{S}_{2} \mathrm{P}(\mathrm{OPr}-\mathrm{n})_{2}\right]_{2}$ & $\begin{array}{c}\text { Deep } \\
\text { Red solid }\end{array}$ & $153^{\circ}$ & $598.54 /(604.61)$ & 6.38/(6.33) & 43.8/(43.7) & 20.88 / (21.21) & 7.80/(7.91) \\
\hline 3 & $\mathrm{Cp}_{2} \mathrm{Ti}\left[\mathrm{S}_{2} \mathrm{P}(\mathrm{OPr}-\mathrm{i})_{2}\right]_{2}$ & $\begin{array}{c}\text { Deep } \\
\text { Red Solid }\end{array}$ & $121^{\circ}$ & $598.69 /(604.61)$ & $6.29 /(6.33)$ & 43.92/(43.70) & $20.98 /(21.21)$ & 7.83/(7.91) \\
\hline 4 & $\mathrm{Cp}_{2} \mathrm{Ti}\left[\mathrm{S}_{2} \mathrm{P}(\mathrm{OBu}-\mathrm{i})_{2}\right]_{2}$ & $\begin{array}{c}\text { Deep } \\
\text { Red Solid }\end{array}$ & $195^{\circ}$ & $659.32 /(660.72)$ & 7.23/(7.01) & 47.16/(47.26) & 18.94 / (19.41) & 6.88/(7.24) \\
\hline 5 & $\mathrm{Cp}_{2} \mathrm{Ti}\left[\mathrm{S}_{2} \mathrm{P}(\mathrm{OPh})_{2}\right]_{2}$ & $\begin{array}{c}\text { Deep } \\
\text { Red Solid }\end{array}$ & $217^{\circ}$ & 738.11/(740.67) & $4.23 /(4.08)$ & $54.89 /(55.13)$ & $16.47 /(17.32)$ & $5.91 /(6.46)$ \\
\hline 6 & $\mathrm{Cp}_{2} \mathrm{Ti}\left[\mathrm{S}_{2} \mathrm{POCH}_{2} \mathrm{CMe}_{2} \mathrm{CH}_{2} \mathrm{O}\right]_{2}$ & $\begin{array}{c}\text { Deep } \\
\text { Red Solid }\end{array}$ & $187^{\circ}$ & $568.67 /(572.52)$ & $5.48 /(5.28)$ & 42.16/(41.95) & 21.97/ (22.40) & 7.95./(8.36) \\
\hline 7 & $\mathrm{Cp}_{2} \mathrm{Ti}\left[\mathrm{S}_{2} \mathrm{POCH}_{2} \mathrm{CEt}_{2} \mathrm{CH}_{2} \mathrm{O}\right]_{2}$ & $\begin{array}{c}\text { Deep } \\
\text { Red Solid }\end{array}$ & $173^{\circ}$ & $627.78 /(628.63)$ & $5.98 /(6.09)$ & $46.22 /(45.85)$ & 19.97 / (20.40) & 7.81./(7.61) \\
\hline 8 & $\mathrm{Cp}_{2} \mathrm{Ti}\left[\mathrm{S}_{2} \mathrm{POCMe}_{2} \mathrm{CMe}_{2} \mathrm{O}\right]_{2}$ & $\begin{array}{c}\text { Deep } \\
\text { Red Solid }\end{array}$ & $203^{\circ}$ & $599.43 /(600.58)$ & $5.98 /(5.70)$ & 43.62/(43.99) & 21.41/ (21.36) & 7.60/(7.97) \\
\hline
\end{tabular}
compounds. 
Table 2. IR spectral data $\left(\mathrm{cm}^{-1}\right)$ of biscyclopentadienyl titanium bis (O,O-dialkyl and alkylene dithiophosphate) compounds.

\begin{tabular}{cccccccc}
\hline SI. No. & Compounds & $v(\mathrm{P})-\mathrm{O}-\mathrm{C}$ & $v \mathrm{P}-\mathrm{O}-(\mathrm{C})$ & Ring Vibration & $v \mathrm{P}=\mathrm{S}$ & $v \mathrm{P}-\mathrm{S}$ & $v(\mathrm{Ti}-\mathrm{S})$ \\
\hline 1 & $\mathrm{Cp}_{2} \mathrm{Ti}\left[\mathrm{S}_{2} \mathrm{P}(\mathrm{OEt})_{2}\right]_{2}$ & $1014.5 \mathrm{~s}$ & $817.8 \mathrm{~s}$ & -- & $644.2 \mathrm{~m}$ & $530.0 \mathrm{w}$ & $400.0 \mathrm{~m}$ \\
2 & $\mathrm{Cp}_{2} \mathrm{Ti}\left[\mathrm{S}_{2} \mathrm{P}(\mathrm{OPr}-\mathrm{n})_{2}\right]_{2}$ & $1060.0 \mathrm{~m}$ & $827.4 \mathrm{~m}$ & -- & $655.8 \mathrm{~m}$ & $520.0 \mathrm{~m}$ & $408.0 \mathrm{w}$ \\
3 & $\mathrm{Cp}_{2} \mathrm{Ti}\left[\mathrm{S}_{2} \mathrm{P}(\mathrm{OPr}-\mathrm{i})_{2}\right]_{2}$ & $1022.2 \mathrm{~m}$ & $800.4 \mathrm{~m}$ & -- & $638.0 \mathrm{~m}$ & $540.0 \mathrm{w}$ & $400.0 \mathrm{~m}$ \\
4 & $\mathrm{Cp}_{2} \mathrm{Ti}\left[\mathrm{S}_{2} \mathrm{P}(\mathrm{OBu}-\mathrm{i})_{2}\right]_{2}$ & $1018.3 \mathrm{~s}$ & $804.3 \mathrm{~s}$ & -- & $661.5 \mathrm{~m}$ & $550.0 \mathrm{~m}$ & $406.0 \mathrm{w}$ \\
5 & $\mathrm{Cp}_{2} \mathrm{Ti}_{2}\left[\mathrm{~S}_{2} \mathrm{P}(\mathrm{OPh})_{2}\right]_{2}$ & $1104.0 \mathrm{~s}$ & $820.8 \mathrm{~s}$ & -- & $682.5 \mathrm{~s}$ & $513.0 \mathrm{w}$ & $409.0 \mathrm{w}$ \\
6 & $\mathrm{Cp}_{2} \mathrm{Ti}\left[\mathrm{S}_{2} \mathrm{POCH}_{2} \mathrm{CMe}_{2} \mathrm{CH}_{2} \mathrm{O}\right]_{2}$ & $10415 \mathrm{~s}$ & $815.8 \mathrm{~m}$ & $987.5 \mathrm{~s}$ & $667.3 \mathrm{~m}$ & $601.7 \mathrm{~m}$ & $410.0 \mathrm{w}$ \\
7 & $\mathrm{Cp}_{2} \mathrm{Ti}\left[\mathrm{S}_{2} \mathrm{POCH}_{2} \mathrm{CEt}_{2} \mathrm{CH}_{2} \mathrm{O}\right]_{2}$ & $1066.6 \mathrm{~s}$ & $937.3 \mathrm{~m}$ & $995.0 \mathrm{~s}$ & $671.2 \mathrm{~m}$ & $602.0 \mathrm{~m}$ & $4280 \mathrm{~m}$ \\
8 & $\mathrm{Cp}_{2} \mathrm{Ti}_{2}\left[\mathrm{~S}_{2} \mathrm{POCMe}_{2} \mathrm{CMe}_{2} \mathrm{O}\right]_{2}$ & $1022.5 \mathrm{~s}$ & $800.4 \mathrm{~s}$ & $921.9 \mathrm{~m}$ & $704.0 \mathrm{~m}$ & $584.4 \mathrm{~m}$ & $414.0 \mathrm{~m}$ \\
\hline
\end{tabular}

$\mathrm{s}=$ strong, $\mathrm{m}$ = medium, $\mathrm{w}=$ weak and $\mathrm{b}=$ broad absorption band.

\section{2. ${ }^{1} \mathrm{H}$ NMR Spectra}

The ${ }^{1} \mathrm{H}$ NMR spectra Biscyclopentadienyl titanium bis (dialkyl and alkylenedithiophosphate) recorded in $\mathrm{CDCl}_{3}$, show the characteristic resonance due to alkoxy and glycoxy (dithio moiety) protons. These ${ }^{1} \mathrm{H}$ NMR spectral data are given in Table 3. The singlet peak at (3.1 - $3.5 \mathrm{ppm})$ in the parent dithiophosphoric acids and assigned to SH proton, is absent from the spectra of Titanium bis (dithiophosphate) derivatives indicating deprotonation of SH group and forming of Ti -S bond [30].

\section{3. ${ }^{13} \mathrm{C}$ NMR Spectra}

The ${ }^{13} \mathrm{C}$ NMR spectra of biscyclopentadienyl titanium bis (dialkyl and alkylenedithiophosphate) complexes were recorded in deuterated chloroform at ambient temperature (Table 4). The spectra show very small chemical shifts when compared to those obtained for the parent dithiophosphoric acids and indicate no substantial difference in the structure [31].

\subsection{P NMR Spectra}

The proton decoupled ${ }^{31} \mathrm{P}$ NMR spectra of biscyclopentadienyl titanium (IV) bis (dialkyl and alkylenedithiophosphate) derivatives, Table 3, show only one signal peak for each complex in the region 77.9 - $93.7 \mathrm{ppm}$. The observation of only one sharp singlet for all compounds reflects the equivalent nature of phosphorous nuclei and the purity of the compound. However, no notable difference was observed in comparison to the parent acids [32] [33]. According to Glidewell, these small shifts indicate monodentate behavior of the ligand [34].

\subsection{Structural Elucidation}

Considering the normal mode of bonding of dithiophosphate with the metal as bidentate chelating ligand and based on the above spectral studies of the complexes using IR, NMR $\left({ }^{1} \mathrm{H},{ }^{13} \mathrm{C},{ }^{13} \mathrm{P}\right)$, molecular weight determination and elemental analyses. We suggest the following structure (Figure 1 and Figure 2).

\subsection{Results of Bioactivity Tests}

The preliminary results show that the chemical compounds slightly inhibited the growth of Escherich coli and Bacillus cereus after a $24 \mathrm{~h}$ incubation period, but had little or no effect on Pseudomonas aeruginosa under similar conditions. See (Table 5) and Figure 3. The results are means of three replicate experiments. Escherichia coli a Gram-negative bacterium commonly found in the lower intestine of warm-blooded organisms [35] appeared to be the most susceptible to all chemicals tested with zones of inhibition ranging from $5.0 \pm 0.76\left(\mathrm{Cp}_{2} \mathrm{Ti}\left[\mathrm{S}_{2} \mathrm{P}(\mathrm{OPr}-\mathrm{n})_{2}\right]_{2}\right)$ to $11.1 \pm 0.54$ for $\left(\mathrm{Cp}_{2} \mathrm{Ti}\left[\mathrm{S}_{2} \mathrm{P}(\mathrm{OEt})_{2}\right]_{2}\right)$. The test chemicals had very little inhibitory effect on Pseudomonas aeruginosa. 


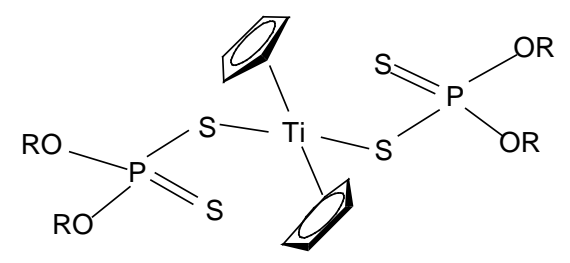

Figure 1. Suggested structure for biscyclopentadienyl titanium bis (dialkyldithiophosphate) derivatives.<smiles></smiles>

Figure 2. Suggested structure for biscyclopentadienyl titanium bis (alkylenedithiophosphate) derivatives.

Table 3. ${ }^{1} \mathrm{H}$ and ${ }^{31} \mathrm{P}$ NMR spectral data of biscyclopentadienyl titanium bis (O,O-dialkyl and alkylene dithiophosphate) compounds.

\begin{tabular}{|c|c|c|c|}
\hline SI. No. & Compounds & ${ }^{1} \mathrm{H}$ chemical shift in $\delta \mathrm{ppm}$ in $\mathrm{CDCl}_{3}$ & ${ }^{31} \mathrm{P}$ chemical (parent acid) \\
\hline 1 & $\mathrm{Cp}_{2} \mathrm{Ti}\left[\mathrm{S}_{2} \mathrm{P}(\mathrm{OEt})_{2}\right]_{2}$ & $\begin{array}{c}1.14, \mathrm{t}(J=6.5 \mathrm{~Hz}), 12 \mathrm{H}\left(\mathrm{CH}_{3}\right), \\
3.7, \mathrm{q}(J=6.0 \mathrm{~Hz}), 8 \mathrm{H}\left(\mathrm{OCH}_{2}\right) \\
6.42, \mathrm{~s}, 10 \mathrm{H}\left(\mathrm{C}_{5} \mathrm{H}_{5}\right)\end{array}$ & 86.0. (85.7) \\
\hline 2 & $\mathrm{Cp}_{2} \mathrm{Ti}\left[\mathrm{S}_{2} \mathrm{P}(\mathrm{OPr}-\mathrm{n})_{2}\right]_{2}$ & $\begin{array}{c}0.76, \mathrm{t}(J=7.5 \mathrm{~Hz}), 12 \mathrm{H}\left(\mathrm{CH}_{3}\right) \\
1.34, \mathrm{~m}(J=6.5 \mathrm{~Hz}), 8 \mathrm{H}\left(\mathrm{CH}_{2}\right) \\
4.0-4.1, \mathrm{t}\left(\mathrm{J}=7.5 \mathrm{~Hz}, 8 \mathrm{H}\left(\mathrm{OCH}_{2}\right)\right. \\
\text { 6.6, s, } 10 \mathrm{H}\left(\mathrm{C}_{5} \mathrm{H}_{5}\right)\end{array}$ & $86.2(86.1)$ \\
\hline 3 & $\mathrm{Cp}_{2} \mathrm{Ti}\left[\mathrm{S}_{2} \mathrm{P}(\mathrm{OPr}-\mathrm{i})_{2}\right]_{2}$ & $\begin{array}{c}1.21, \mathrm{~d}(J=6.6 \mathrm{~Hz}), 24 \mathrm{H}\left(\mathrm{CH}_{3}\right) \\
4.40-4.42, \mathrm{~m} J(\mathrm{PH})=12 \mathrm{~Hz}, 4 \mathrm{H}(\mathrm{OCH}) \\
6.42, \mathrm{~s}, 10 \mathrm{H}\left(\mathrm{C}_{5} \mathrm{H}_{5}\right)\end{array}$ & $82.3(82.3)$ \\
\hline 4 & $\mathrm{Cp}_{2} \mathrm{Ti}\left[\mathrm{S}_{2} \mathrm{P}(\mathrm{OBu}-\mathrm{i})_{2}\right]_{2}$ & $\begin{array}{c}0.8, \mathrm{~d}(J=7 \mathrm{~Hz}), 24 \mathrm{H}\left(\mathrm{CH}_{3}\right) \\
1.92, \mathrm{~m}(J=6.5 \mathrm{~Hz}), 4 \mathrm{H}(\mathrm{CH}) \\
3.80, \mathrm{~d}(J=7 \mathrm{~Hz}), 8 \mathrm{H}\left(\mathrm{OCH}_{2}\right) \\
\text { 6.60, s, } 10 \mathrm{H}\left(\mathrm{C}_{5} \mathrm{H}_{5}\right)\end{array}$ & $85.6(85.7)$ \\
\hline 5 & $\mathrm{Cp}_{2} \mathrm{Ti}\left[\mathrm{S}_{2} \mathrm{P}(\mathrm{OPh})_{2}\right]_{2}$ & $\begin{array}{c}7.2 \text { - 7.4, m, } 2 \mathrm{OH}\left(\mathrm{OC}_{6} \mathrm{H}_{5}\right) \\
6.4, \mathrm{~s}, 10 \mathrm{H}\left(\mathrm{C}_{5} \mathrm{H}_{5}\right)\end{array}$ & 79.9 (79.9) \\
\hline 6 & $\mathrm{Cp}_{2} \mathrm{Ti}\left[\mathrm{S}_{2} \mathrm{POCH}_{2} \mathrm{CMe}_{2} \mathrm{CH}_{2} \mathrm{O}\right]_{2}$ & $\begin{array}{c}0.82, \mathrm{~s}, 12 \mathrm{H}\left(\mathrm{CH}_{3}\right) \\
\text { 4.10, d, } 8 \mathrm{H}\left(\mathrm{OCH}_{2}\right), J\left(\mathrm{PH}^{2}\right)=15.6 \mathrm{~Hz} \\
\text { 6.35, s, } 10 \mathrm{H}\left(\mathrm{C}_{5} \mathrm{H}_{5}\right)\end{array}$ & $77.4(77.3)$ \\
\hline 7 & $\mathrm{Cp}_{2} \mathrm{Ti}\left[\mathrm{S}_{2} \mathrm{POCH}_{2} \mathrm{CEt}_{2} \mathrm{CH}_{2} \mathrm{O}\right]_{2}$ & $\begin{array}{c}0.71, \mathrm{t}(\mathrm{J}=7.5 \mathrm{~Hz}), 12 \mathrm{H}\left(\mathrm{CH}_{3}\right) \\
1.11, \mathrm{q}(\mathrm{J}=7.5 \mathrm{~Hz}), 8 \mathrm{H}\left(\mathrm{CH}_{2}\right) \\
\text { 4.02, d, 8H(OCH}), J(\mathrm{PH})=16 \mathrm{~Hz} \\
6.28, \mathrm{~s}, 10 \mathrm{H}\left(\mathrm{C}_{5} \mathrm{H}_{5}\right)\end{array}$ & $78.3(78.5)$ \\
\hline 8 & $\mathrm{Cp}_{2} \mathrm{Ti}\left[\mathrm{S}_{2} \mathrm{POCMe}_{2} \mathrm{CMe}_{2} \mathrm{O}\right]_{2}$ & $\begin{array}{l}1.06, \mathrm{~s}, 24 \mathrm{H}\left(\mathrm{CH}_{3}\right) \\
6.50, \mathrm{~s}, 10 \mathrm{H}\left(\mathrm{C}_{5} \mathrm{H}_{5}\right)\end{array}$ & $93.4(93.1)$ \\
\hline
\end{tabular}

Table 4. ${ }^{13} \mathrm{C}$ NMR spectral data of biscyclopentadienyl titanium bis (O,O-dialkyl and alkylene dithiophosphate.

\begin{tabular}{|c|c|c|c|c|c|c|c|}
\hline \multirow{2}{*}{ SI. No. } & \multirow{2}{*}{ Compound } & \multicolumn{6}{|c|}{${ }^{13} \mathrm{C}$ Chemical shift, in ppm } \\
\hline & & $\mathrm{CH}_{3}$ & $\mathrm{CH}_{2}$ & $\mathrm{CH}$ & $\mathrm{C}$ & $\mathrm{CO}$ & $\mathrm{C}_{5} \mathrm{H}_{5}$ \\
\hline 2 & $\mathrm{Cp}_{2} \mathrm{Ti}\left[\mathrm{S}_{2} \mathrm{P}(\mathrm{OPr}-\mathrm{n})_{2}\right]_{2}$ & $10.0 \mathrm{~s}$ & $23.0 \mathrm{~s}$ & & & $70.2 \mathrm{~s}$ & $120.4 \mathrm{~s}$ \\
\hline 4 & $\mathrm{Cp}_{2} \mathrm{Ti}\left[\mathrm{S}_{2} \mathrm{P}(\mathrm{OBu}-\mathrm{i})_{2}\right]_{2}$ & $18.8 \mathrm{~s}$ & $28.5 \mathrm{~s}$ & & & $74.2 \mathrm{~s}$ & $120.4 \mathrm{~s}$ \\
\hline 7 & $\mathrm{Cp}_{2} \mathrm{Ti}\left[\mathrm{S}_{2} \mathrm{POCH}_{2} \mathrm{CEt}_{2} \mathrm{CH}_{2} \mathrm{O}\right]_{2}$ & $6.8 \mathrm{~s}$ & $22.2 \mathrm{~s}$ & & $36.9 \mathrm{~s}$ & $76.2 \mathrm{~s}$ & $120.7 \mathrm{~s}$ \\
\hline
\end{tabular}




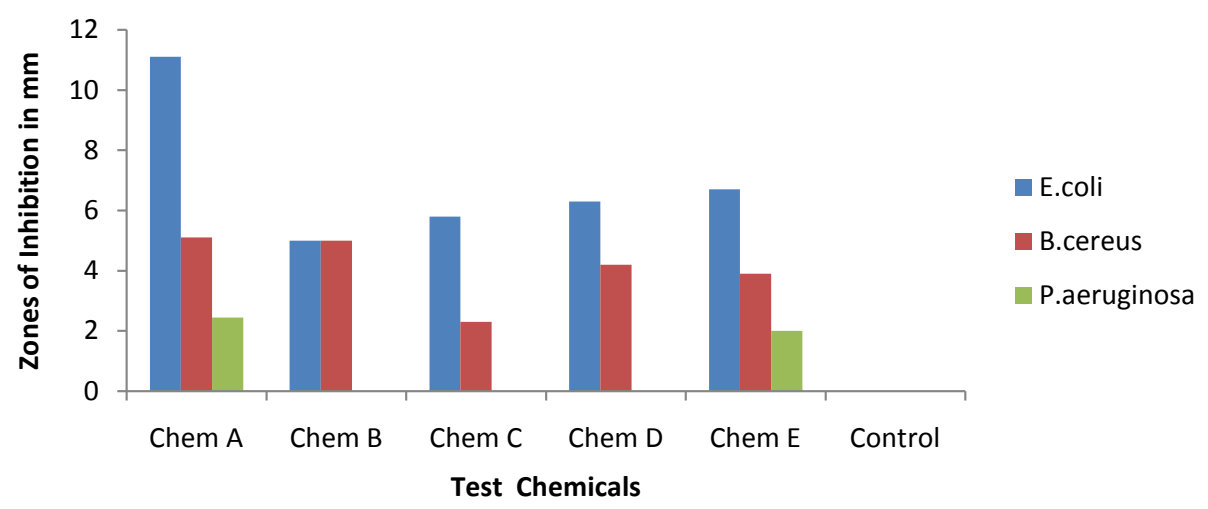

Figure 3. Antimicrobial activity of test chemicals.

Table 5. Zones of inhibition (mm) of test chemicals against E. coli, B. cereus and P. aeruginosa.

\begin{tabular}{|c|c|c|c|}
\hline TEST chem. compounds & Escherichia coli & Bacillus cereus & Pseudomonas aeruginosa \\
\hline $\mathrm{Cp}_{2} \mathrm{Ti}\left[\mathrm{S}_{2} \mathrm{P}(\mathrm{OEt})_{2}\right]_{2}$ & $11.1 \pm 0.54$ & $5.1 \pm 0.32$ & $2.45 \pm 1.5$ \\
\hline $\mathrm{Cp}_{2} \mathrm{Ti}\left[\mathrm{S}_{2} \mathrm{P}(\mathrm{OPr}-\mathrm{n})_{2}\right]_{2}$ & $5.0 \pm 0.76$ & $5.0 \pm 0.78$ & 0.00 \\
\hline $\mathrm{Cp}_{2} \mathrm{Ti}\left[\mathrm{S}_{2} \mathrm{P}(\mathrm{OPr}-\mathrm{i})_{2}\right]_{2}$ & $5.8 \pm 0.81$ & $2.3 \pm 0.21$ & 0.00 \\
\hline $\mathrm{Cp}_{2} \mathrm{Ti}\left[\mathrm{S}_{2} \mathrm{P}(\mathrm{OBu}-\mathrm{i})_{2}\right]_{2}$ & $6.3 \pm 0.41$ & $4.2 \pm 0.46$ & 0.00 \\
\hline $\mathrm{Cp}_{2} \mathrm{Ti}\left[\mathrm{S}_{2} \mathrm{P}\left(\mathrm{OCH}_{2} \mathrm{C}(\mathrm{Et})_{2} \mathrm{CH}_{2} \mathrm{O}\right)_{2}\right]_{2}$ & $6.7 \pm 0.72$ & $3.9 \pm 0.18$ & $2.00 \pm 3.0$ \\
\hline
\end{tabular}

\section{Conclusion}

We have successfully synthesized and characterized the new biscyclopentadienyl titanium (IV) bis (O,O dialkyl and alkylenedithiophosphate) compounds. The molecular weight of all these products determined by cryoscopic method in benzene indicated the monomeric nature of these products. The IR, ${ }^{1} \mathrm{H},{ }^{13} \mathrm{C}$ and ${ }^{31} \mathrm{P}$ NMR spectra and the elemental analysis of all of these titanium complexes are consistent with the proposed tentative structure: (see Figure 1 and Figure 2). These novel chemical compounds used in this study show monodentate bond with the metal center and antimicrobial activity against $E$. coli and B. cereus and weak or no effect on $P$. aeruginosa.

\section{Acknowledgements}

The authors are thankful for financial support from Evans-Allen Federal Appropriated Funds. The authors also wish to express their profound appreciation for the support received from Dr. L. Walker, Prof. Matthew Edwards, Dean Chance M. Glenn, Ms. Dianne Kirnes and Shonda Scott.

\section{References}

[1] Ma, N., Li, Y., Xu, H., Wang, Z. and Zhang, X. (2010) Well-Defined, Reversible Boronate Crosslinked Nanocarriers for Targeted Drug Delivery in Response to pH and cis-Diols. Journal of the American Chemical Society, 132, 442-443.

[2] El khaldy, A.A.S., Abushanab, A.M. and Abu Alkhair, E. (2011) Synthesis and Antimicrobial Studies of Bis (O,O'Dialkyl and Alkylene Dithiophosphoric Acids) Adducts of Diphenyl Diselenide. Applied Organometallic Chemistry, 25, 487-595.

[3] Maheshwari, S., Drake, J.E., Kori, K., Light, M.E. and Ratnani, R. (2009) Synthesis and Spectroscopic Characterization of Tris(O,O'-Ditolyldithiophosphato) Arsenic/Antimony/Bismuth(III) Compounds: Crystal Structures of $\left[\right.$ As $\left\{\mathrm{S}_{2} \mathrm{P}\right.$ $\left.\left.\left(\mathrm{OC}_{6} \mathrm{H}_{4} \mathrm{Me}-\mathrm{m}\right)(2)\right\}(3)\right]$ Center Dot 0.5C(6)H(14), $\left[\mathrm{Sb}\left\{\mathrm{S}_{2} \mathrm{P}\left(\mathrm{OC}_{6} \mathrm{H}_{4} \mathrm{Me}-\mathrm{m}\right)(2)\right\}(3)\right]$ and $\left[\mathrm{Bi}\left\{\mathrm{S}_{2} \mathrm{P}\left(\mathrm{OC}_{6} \mathrm{H}_{4} \mathrm{Me}-\mathrm{m}\right)(2)\right\}(3)\right]$. Polyhedron, 28, 689-694.

[4] Bingham, A.L., Drake, J.E., Hursthouse, M.B., Light, M.E., Nirwan, M. and Ratnani, R. (2007) Synthesis, Characterization and Spectral Studies of Nitrogen Base Adducts of bis(O,O'-Ditolyldithiophosphato)Nickel(II). Crystal Structures of $\mathrm{Ni}\left[\mathrm{S}_{2} \mathrm{P}\left(\mathrm{OC}_{6} \mathrm{H}_{4} \mathrm{Me}-p\right)_{2}\right]_{2} \cdot \mathrm{C}_{10} \mathrm{H}_{8} \mathrm{~N}_{2}$ and $\mathrm{Ni}\left[\mathrm{S}_{2} \mathrm{P}\left(\mathrm{OC}_{6} \mathrm{H}_{4} \mathrm{Me}-\mathrm{o}\right)_{2}\right]_{2} \cdot \mathrm{C}_{14} \mathrm{H}_{12} \mathrm{~N}_{2} \cdot \mathrm{C}_{6} \mathrm{H}_{6}$. Polyhedron, 26, $2672-2678$.

[5] Cotero-Villegas, A.M., Toscano, R.-A., Muñoz-Hernándeza, M., López-Cardosoa, M, García y Garcíaa, P. and Cea- 
Olivares, R. (2005) Synthesis, Spectroscopic Characterization of O,O-Alkylene Dithiophosphates of Tellurolane and 1Oxa-4-Tellurane. Single Crystal Structures of $\mathrm{C}_{4} \mathrm{H}_{8} \mathrm{Te}\left[\mathrm{S}_{2} \mathrm{P}\left(\mathrm{OCH}_{2}\right)_{2} \mathrm{CMe}-\mathrm{nPr}\right]_{2}$ and $\mathrm{C}_{4} \mathrm{H}_{8} \mathrm{OTe}\left[\mathrm{S}_{2} \mathrm{P}\left(\mathrm{OCH}_{2}\right)_{2} \mathrm{CEt}_{2}\right]_{2}$. Journal of Organometallic Chemistry, 690, 2872-2879. http://dx.doi.org/10.1016/j.jorganchem.2005.01.058

[6] Dave, G.V. and Vyas, P.J. (2012) Synthesis, Structural Elucidation and Antimicrobial Activities of Some Alkylene Dithiophosphate Derivatives of Macrocyclic Complexes of Ni (II) Having $\mathrm{N}_{2} \mathrm{~S}_{2}$ Potential Donors in 18 to 24 Membered Rings. Journal of Current Chemical \& Pharmaceutical Sciences, 2, 133-148.

[7] El khaldy, A.A., Hussien, A.R., Abushanab, A.M. and Wasse, M.A. (2011) Synthesis and Characterization of ChloroBis (Cyclopentadienyl) Titanium(IV) and Zirconium(IV) O,O'-Dialkyl and Alkylene Dithiophosphates. Phosphorus, Sulfur, and Silicon and the Related Elements, 186, 589-597.

[8] Yadav, J.S., Mehrotra, R.K. and Srivastava, G. (1987) Metal and Organometal Complexes of Oxy- and Thio-Phosphorus Acids-I. O,O-Alkylene and Dialkyl Dithiophosphates of Titanium(IV). Polyhedron, 6, 1687-1693. http://dx.doi.org/10.1016/S0277-5387(00)80772-X

[9] Chauhan, H.P.S., Singh, U.P., Shaik, N.M., Mathur, S. and Huch, V. (2006) Synthetic, Spectroscopic, X-Ray Structural and Antimicrobial Studies of 1,3-Dithia-2-Stibacyclopentane Derivatives of Phosphorus Based Dithiolato Ligands. Polyhedron, 25, 2841-2847. http://dx.doi.org/10.1016/j.poly.2006.04.027

[10] Drake J.E., Gurnani, G., Hursthouse, M.B., Light, M.E., Nirwan, M. and Ratnani, R. (2007) Synthesis and Spectroscopic Characterization of Dimethyl/di(n-butyl)tin(IV)bis(O,O'-Ditolyl Dithiophosphate) Complexes. Crystal Structures of $\mathrm{Me}_{2} \mathrm{Sn}\left[\mathrm{S}_{2} \mathrm{P}\left(\mathrm{OC}_{6} \mathrm{H}_{4} \mathrm{Me}-\mathrm{o}\right)_{2}\right]_{2}$ and $n-\mathrm{Bu}_{2} \mathrm{Sn}\left[\mathrm{S}_{2} \mathrm{P}\left(\mathrm{OC}_{6} \mathrm{H}_{4} \mathrm{Me}-\mathrm{o}\right)_{2}\right]_{2}$. Applied Organometallic Chemistry, 2, 539-544. http://dx.doi.org/10.1002/aoc.1265

[11] Chauhan, H.P.S. and Singh, U.P. (2007) Synthetic, Spectral, Thermal and Antimicrobial Studies on Some Bis( $N, N$ 'Dialkyldithiocarbamato) Antimony(III) Alkylenedithiophosphates. Applied Organometallic Chemistry, 21, 880-889. http://dx.doi.org/10.1002/aoc.1290

[12] Sharma, P.K., Rehwani, H., Gupta, R.S. and Singh, Y.P. (2007) The Antispermatogenic Activity of Some Phenylbismuth(III) O,O'-Dialkyldithiophosphates. Applied Organometallic Chemistry, 21, 701-710. http://dx.doi.org/10.1002/aoc.1238

[13] Shah, F.U., Glavatskih, S., Höglund, E., Lindberg, M. and Antzutkin, O.N. (2011) Interfacial Antiwear and Physicochemical Properties of Alkylborate-Dithiophosphates. ACS Applied Materials \& Interfaces, 3, 956-968. http://dx.doi.org/10.1021/am101203t

[14] Juliano, R.C., Curtius, A.G. and Borges, D.L.G. (2012) Diethyldithiophosphate (DDTP): A Review on Properties, General Applications, and Use in Analytical. Applied Spectroscopy Reviews, 47, 583-619. http://dx.doi.org/10.1080/05704928.2012.682286

[15] Lee, Y.A., McGarrah, J.F., Lachicotte, R.J. and Eisenberg. R. (2002) Multiple Emissions and Brilliant White Luminescence from Gold(I) O,O'-Di(alkyl)Dithiophosphate Dimers. Journal of the American Chemical Society, 124, 1066210663. http://dx.doi.org/10.1021/ja0267876

[16] Margielewski, L. (2010) The Effect of Zinc Dithiophosphates on Friction and Wear of Partially Stabilized Zirconia. Part I, Zinc Di-n-alkyldithiophosphates Tribological Properties. Tribologia, 230, 87-104.

[17] Hernandez-Molina, R., Gonzalez-Platas, J., Kovalenko, K.A., Sokolov, M.N., Virovets, A.V., Llusar, R. and Vicent, C. (2011) Cuboidal $\mathrm{Mo}_{3} \mathrm{~S}_{4}$ and $\mathrm{Mo}_{3} \mathrm{NiS}_{4}$ Complexes Bearing Dithiophosphates and Chiral Carboxylate Ligands: Synthesis, Crystal Structure and Fluxionality. European Journal of Inorganic Chemistry, 5, 683-693. http://dx.doi.org/10.1002/ejic.201000795

[18] Klapoetke, T.M., Koepf, H., Tornieporth-Oetting, I.C. and White, P.S. (1994) Synthesis, Characterization, and Structural Investigation of the First Bioinorganic Titanocene(IV).alpha.-Amino Acid Complexes Prepared from the Antitumor Agent Titanocene Dichloride. Organometallics, 13, 3628-3633. http://dx.doi.org/10.1021/om00021a040

[19] Clearfield, A., Warner, D.K., Saldarriaga-Molina, C.H., Ropal, R. and Bernal, I. (1975) Structural Studies of $\left(\pi-C_{5} H_{5}\right)_{2} M_{2}$ Complexes and their Derivatives. The Structure of Bis $(\pi$-cyclopentadienyl)titanium Dichloride. Canadian Journal of Chemistry, 53, 1622-1629. http://dx.doi.org/10.1139/v75-228

[20] Chauhan, H.P.S., Bhasin, C.P.G., Srivastava, G. and Mehrotra, R.C. (1983) Synthesis and Characterization of 2-Mercapto-2-Thiono-1,3,2-Dioxaphospholanes and Dioxaphosphorinanes. Phosphorus and Sulfur and the Related Elements, 15, 99-104. http://dx.doi.org/10.1080/03086648308073283

[21] Clinical and Laboratory Standards Institute (2012) Approved Standard CLSI Document M07-A9. 9th Edition, Clinical and Laboratory Standards Institute, Wayne.

[22] Kato, S., Hori, A., Shiotani, H., Mizuta, M., Hayashi, N. and Takakuwa, T. (1974) Infrared and Raman Spectra of (Thioacetoxythio)Triorgano Derivatives of Silicon, Germanium, Tin and Lead. Journal of Organometallic Chemistry, 82, 223-228. http://dx.doi.org/10.1016/S0022-328X(00)90359-0

[23] Sowerby, D.B., Haiduc, I., Barbul-Rusu, A. and Salajan. M. (1983) Antimony(III) Diorganophosphoro- and Diorga- 
nophospinodithioates: Crystal Structure of $\mathrm{Sb}\left[\mathrm{S}_{2} \mathrm{P}(\mathrm{OR})_{2}\right]_{3}(\mathrm{R}=\mathrm{Me}$ and i-Pr). Inorganica Chimica Acta, 162, 87-96. http://dx.doi.org/10.1016/S0020-1693(00)88943-4

[24] Pavia, D.L., Lampman, G.M. and Kris, G.S. (1996) Introduction to Spectroscopy. 2nd Edition, Saunders Golden Sunburst Series, Orlando.

[25] Corbridge, D.E.C. (1969) Infra-Red Spectra of Phosphorus Compounds. Topics in Phosphorus Chemistry, 6, $235-366$.

[26] Ohkaku, N. and Nakamoto, N. (1973) Metal Isotope Effect on Metal-Liquid Vibrations. X. Far-Infrared Spectra of Trans Adducts of Tin(IV) Tetrahalide with Unidentate Ligands. Inorganic Chemistry, 12, 2440-2446. http://dx.doi.org/10.1021/ic50128a043

[27] Lockhart, T.P. and Manders, W.P. (1986) Structure Determination by NMR Spectroscopy. Dependence of |2J(119Sn,1H)| on the Me-Sn-Me Angle in Methyltin(IV) Compounds. Inorganic Chemistry, 25, 892-895. http://dx.doi.org/10.1021/ic00227a002

[28] Drew, M.G.B., Baricalli, P.J., Mitchell, P.C.H. and Read, A.R. (1983) Crevice Co-Ordination: Binding of a Ligand Molecule in a Molecular Crevice. Crystal and Molecular Structures of $\mu$-Oxo- $\mu$-pyridine- $\mu$-sulphido-bis[(OO'-di-isopropyl phosphorodithioato)oxo-molybdenum(V)] and $\mu$-Oxo- $\mu$-pyridazine- $\mu$-sulphido-bis[(OO'-di-isopropyl phosphorodithioato)oxomolybdenum(V)]. Journal of the Chemical Society, Dalton Transactions, 649-655. http://dx.doi.org/10.1039/dt9830000649

[29] Gupta, R.K., Rai, A.K., Mehrotra, R.C. and Jain, V.K. (1984) Cyclic O,O-Alkylenedithiophosphates of Phenyl-Arsenic and -Antimony. Inorganica Chimica Acta, 88, 201-207. http://dx.doi.org/10.1016/S0020-1693(00)83597-5

[30] Gupta, R.K., Rai, A.K., Mehrotra, R.C., Jain, V.K., Hoskins, P.F. and Tiekink, E.R.T. (1985) Phenylarsenic(III) and Phenylantimony(III) Bis(dialkyl dithiophosphates): Synthesis and Multinuclear (Proton, Carbon-13, Phosphorus-31) NMR and Mass Spectral Studies. Crystal and Molecular Structures of $\mathrm{C}_{6} \mathrm{H}_{5} \mathrm{M}\left[\mathrm{S}_{2} \mathrm{P}(\mathrm{OCHMe})_{2}\right]_{2}[\mathrm{M}=\mathrm{Sb}(\mathrm{III})$ and $\mathrm{As}(\mathrm{III})]$. Inorganic Chemistry, 24, 3280-3284. http://dx.doi.org/10.1021/ic00214a037

[31] Srivastava, S.K., Tomar, S., Rastogi, R. and Saxena, R. (2010) Substituted Diorganotin(IV) O,O'-Alkylene Dithiophosphates: Synthesis and Spectral Aspects. Phosphorus, Sulfur, and Silicon and the Related Elements, 185, 634-640. http://dx.doi.org/10.1080/10426500902894959

[32] Chauhan, H.P.S. (1998) Chemistry of Diorganodithiophosphate (and Phosphinate) Derivatives with Arsenic, Antimony and Bismuth. Coordination Chemistry Reviews, 173, 1-30. http://dx.doi.org/10.1016/S0010-8545(97)00071-4

[33] Chauhan, H.P.S. (2003) The Chemistry and Applications of Alkoxy, Aryloxy and Applied Derivatives of Elements. RBSA Publishers, Jaipur.

[34] Glidewell, C. (1977) Ambident Nucleophiles: VI. Solution Metal-Ligand Binding Modes in Phosphorodithioate Complexes. A Phosphorus-31 N.M.R. Study. Inorganica Chimica Acta, 25, 159-163. http://dx.doi.org/10.1016/S0020-1693(00)95706-2

[35] Abu-Basha, E.A., Gharaibeh, S.M. and Thabet, A.M. (2012) In Vitro Susceptibility of Resistant, Escherichia coli Field Isolates to Antimicrobial Combinations. The Journal of Applied Poultry Research, 21, 595-602. http://dx.doi.org/10.3382/japr.2011-00500 
Scientific Research Publishing (SCIRP) is one of the largest Open Access journal publishers. It is currently publishing more than 200 open access, online, peer-reviewed journals covering a wide range of academic disciplines. SCIRP serves the worldwide academic communities and contributes to the progress and application of science with its publication.

Other selected journals from SCIRP are listed as below. Submit your manuscript to us via either submit@scirp.org or Online Submission Portal.
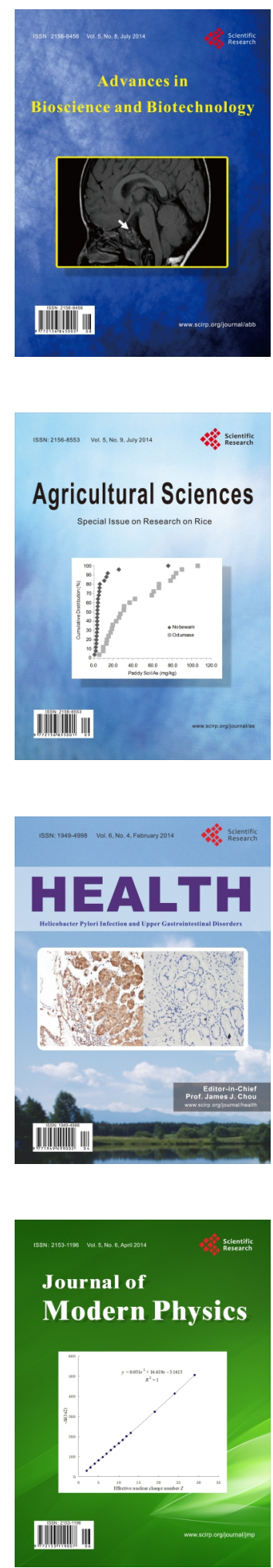
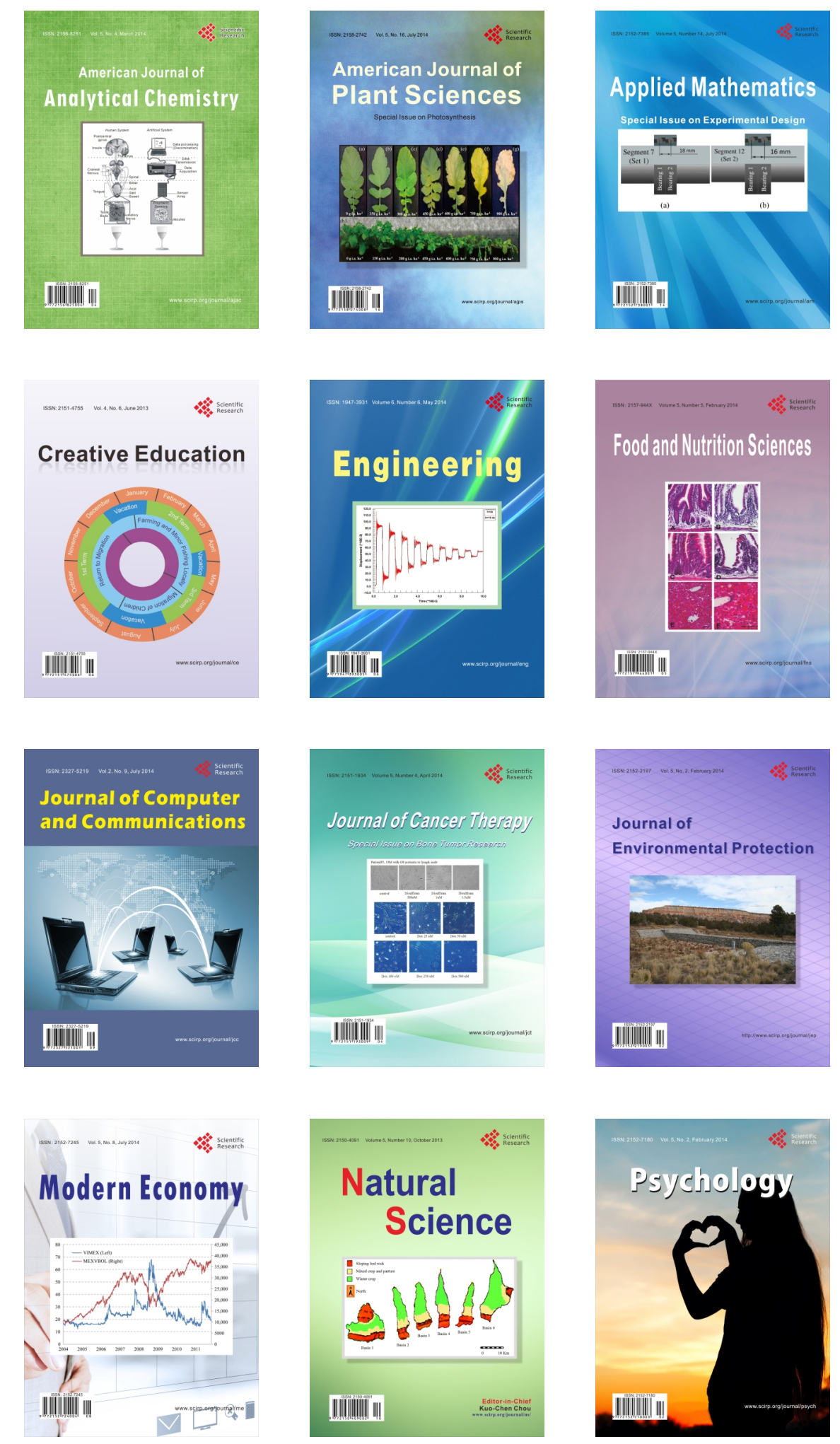